\title{
Ru Nanoparticles Supported on MIL-101 by Double Solvents Method as High-Performance Catalysts for Catalytic Hydrolysis of Ammonia Borane
}

\author{
Tong Liu, ${ }^{1}$ Qingtao Wang, ${ }^{1}$ Bingzheng Yan, ${ }^{1}$ Mei Zhao, ${ }^{1}$ Wenbo Li, ${ }^{1}$ and Haiyan Bie ${ }^{2}$ \\ ${ }^{1}$ College of Materials Science and Engineering, Qingdao University of Science and Technology, Qingdao, Shandong 266000, China \\ ${ }^{2}$ College of Chemistry and Chemical Engineering, Ocean University of China, Qingdao, Shandong 266000, China \\ Correspondence should be addressed to Haiyan Bie; haiyanbie@ouc.edu.cn
}

Received 10 July 2015; Accepted 10 August 2015

Academic Editor: Jijeesh R. Nair

Copyright (C) 2015 Tong Liu et al. This is an open access article distributed under the Creative Commons Attribution License, which permits unrestricted use, distribution, and reproduction in any medium, provided the original work is properly cited.

\begin{abstract}
Highly dispersed crystalline Ru nanoparticles (NPs) were successfully immobilized inside the pores of MIL-101 by a double solvents method (DSM). HRTEM clearly demonstrated the uniform distribution of the ultrafine Ru NPs throughout the interior cavities of MIL-101. The synthesized Ru@MIL-101 catalyst was also characterized by X-ray diffraction (XRD), $\mathrm{N}_{2}$ adsorption desorption, and ICP-AES. The catalytic test indicated that the Ru NPs supported MIL-101 material exhibited exceedingly high activity and excellent durability for hydrogen generation from the catalytic hydrolysis of amine boranes.
\end{abstract}

\section{Introduction}

Renewable and clean energy is believed as a long-term solution to replace the current prevalent petroleum energy. Recently, hydrogen receives much attention as a nextgeneration energy carrier [1-3]. Amine boranes $\left(\mathrm{NH}_{3}-\mathrm{BH}_{3}\right.$, $\mathrm{AB}$ ) appears to be a suitable hydrogen source, because of its rich hydrogen content, high stability, and nontoxicity [4-7]. The hydrogen stored in $\mathrm{AB}$ can be released through the hydrolysis of $\mathrm{AB}$ in the presence of a suitable catalyst according to the reaction (1). So far, various catalysts have been developed for the catalytic hydrolysis of $\mathrm{AB}$ to generate hydrogen, such as Pt-based catalyst [8] and Ru-based catalyst [9]. Ru-based catalyst showed significant advantages in hydrogen generation from $\mathrm{AB}$. However, the balance between costs, efficiency, and recyclability still remains a considerable challenge:

$$
\begin{aligned}
& \mathrm{NH}_{3}-\mathrm{BH}_{3}(\mathrm{aq})+2 \mathrm{H}_{2} \mathrm{O}(\mathrm{I}) \\
& \stackrel{\text { catalyst }}{\longrightarrow} \mathrm{NH}_{4} \mathrm{BO}_{2}(\mathrm{aq})+3 \mathrm{H}_{2}(\mathrm{~g})
\end{aligned}
$$

Porous metal-organic frameworks (MOFs) have emerged as a class of promising functional porous materials, especially in the applications of gas storage catalysis, separation, sensing, and drug delivery [10-13]. Taking advantages of their high specific surface area and tunable pore size, efficient solid catalysts can be achieved by loading MNPs inside the porous MOFs. It is reported that two approaches are used for the synthesis of MNPs inside MOFs. The first and most widely used approach is to use MOFs as stabilizing host material, which provides a confined space for nucleation, such as chemical vapor deposition [14], solution infiltration [1517], and solid grinding [18]. However, it seems difficult to completely avoid the precursor compounds and product aggregation on the external surface of MOFs. Therefore, a double solvents method (DSM) has been reported to incorporate fine metal nanoparticles within the pores of MOFs in the absence of NPs aggregation on the external surface of framework. Until now, few cases concerning double solvents method were investigated in the synthesis of MOFs-based catalysts for catalytic hydrolysis of ammonia borane $[19,20]$.

In this work, the highly dispersed Ru NPs were successfully encapsulated in the pores of MIL-101 without aggregation on the external surfaces of the host framework by DSM (Figure 1). MIL-101, a chromium-based MOF, was chosen as a host matrix because of its high stability in water, extralarge surface area, and two kinds of hydrophilic cavities 


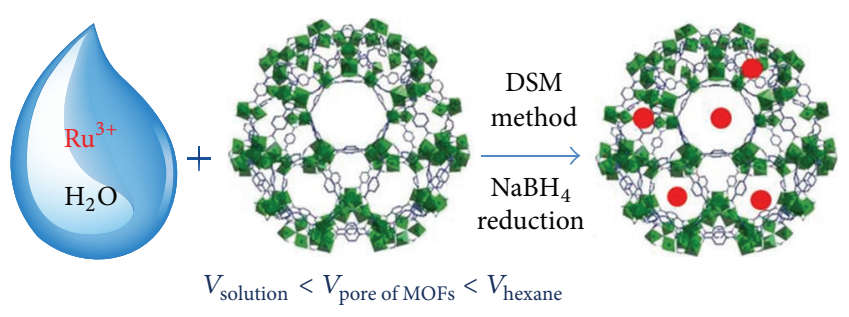

$\mathrm{Ru}$

FIGURE 1: Schematic representation of immobilization of the Ru nanoparticles by the MIL-101 matrix using the DSM.

with free diameters of ca. 2.9 and $3.4 \mathrm{~nm}$ accessible through two pore windows of ca. 1.2 and $1.6 \mathrm{~nm}$ in diameter. The metal precursor, $\mathrm{RuCl}_{3}$, can easily diffuse into the internal cavities. Therefore, a hydrophilic solvent (water) containing the metal precursor with a volume set equal to or less than the pore volume of the adsorbent and a hydrophobic solvent (hexane), which was unable to drive through pore windows, were used for preparing $\mathrm{Ru}$ NPs encapsulated by MIL101. The catalytic activity for hydrolytic dehydrogenation of AB of Ru@MIL-101 was investigated. Compared with other reported Ru-based catalysts by liquid impregnation method [21] and heterogeneous Ru(III) catalysts [22], the Ru@MIL101 exhibits remarkably enhanced catalytic activity.

\section{Experimental}

2.1. Chemicals and Materials. All chemicals were commercial and used without further purification. Sodium borohydride $\left(\mathrm{NaBH}_{4},>96 \%\right.$, Sinopharm Chemical Reagent Co., Ltd.), ruodium chloride hydrate $\left(\mathrm{RuCl}_{3} \cdot 3 \mathrm{H}_{2} \mathrm{O}, 38.0-42.0 \%\right.$, Aladdin Industrial Inc.), terephthalic acid $\left(\mathrm{HOOCC}_{6} \mathrm{H}_{4} \mathrm{COOH}\right.$, 99\%, Aladdin Industrial Inc.), chromic nitrate nonahydrate $\left(\mathrm{Cr}\left(\mathrm{NO}_{3}\right)_{3} \cdot 9 \mathrm{H}_{2} \mathrm{O}, 99 \%\right.$, Aladdin Industrial Inc.), hydrochloric acid ( $\mathrm{HCl}, 37 \%$, Sinopharm Chemical Reagent Co., Ltd.), hydrofluoric acid solution (HF, 40\%, Sinopharm Chemical Reagent Co., Ltd.), anhydrous $n$-hexane (Dikma Technologies Inc.), sodium hydroxide $(\mathrm{NaOH}>96 \%$, Sinopharm Chemical Reagent Co., Ltd.), and ethanol $\left(\mathrm{C}_{2} \mathrm{H}_{5} \mathrm{OH}, 99 \%\right.$, Sinopharm Chemical Reagent Co., Ltd.,) were used as received.

2.2. Synthesis of MIL-101. MIL-101 were synthesized according to the reported procedure [23]. Terephthalic acid (0.823 g, $5.0 \mathrm{mmol}), \mathrm{Cr}\left(\mathrm{NO}_{3}\right)_{3} \cdot 9 \mathrm{H}_{2} \mathrm{O}(2.007 \mathrm{mg}, 5.0 \mathrm{mmol})$, HF solution $(1 \mathrm{~mL}, 5.0 \mathrm{mmol})$, and deionized water $(24 \mathrm{~mL})$ were reacted in a $100 \mathrm{~mL}$ Teflon-liner autoclave at $220^{\circ} \mathrm{C}$ for $8 \mathrm{~h}$. After cooling, the resulting green solution of MIL-101 can be isolated from the residual needle-shaped terephthalic acid by using a large pore fritted glass filter $(100 \mu \mathrm{m})$. The free terephthalic acid was eliminated using a small pore fritted glass filter $(30 \mu \mathrm{m})$. The powder was further purified by solvothermal treatment in ethanol at $80^{\circ} \mathrm{C}$ for $24 \mathrm{~h}$. To eliminate the terephthalic acid inside the pores of MIL-101, the resulting green solid was soaked in $\mathrm{NH}_{4} \mathrm{~F}(1 \mathrm{M})$ solution at $70^{\circ} \mathrm{C}$ for
$24 \mathrm{~h}$ and immediately filtered, washed with hot water several times, and finally dried overnight at $150^{\circ} \mathrm{C}$ under vacuum.

2.3. Synthesis of Ru@MIL-101. Supported Ru nanoparticles were prepared via a double solvents method. Typically, $100 \mathrm{mg}$ of activated MIL-101 was suspended in $20 \mathrm{~mL}$ of dry $n$-hexane as hydrophobic solvent and the mixture was sonicated for $15 \mathrm{~min}$ until it became homogeneous. After stirring for $2 \mathrm{~h}, 0.2 \mathrm{~mL}$ of aqueous $\mathrm{HCl}$ solution $(1 \mathrm{~mol} / \mathrm{L}$ ) with different $\mathrm{RuCl}_{3}$ concentrations as the hydrophilic solvent was added dropwise under continuous vigorous stirring during 15 minutes. The resulting solution was continuously stirred for $2 \mathrm{~h}$. After filtration, the green powder was dried under vacuum at 50 C. Ru@MIL-101 catalysts were obtained by reduction with $3.5 \mathrm{~mL}$ freshly prepared aqueous $\mathrm{NaBH}_{4}$ solution $(0.6 \mathrm{M})$ and subsequent centrifugation.

2.4. Catalytic Activity Investigations. In a typical experiment, Ru@MIL-101 powders (50 mg) were dispersed in $4 \mathrm{~mL}$ of water kept in a $30 \mathrm{~mL}$ of two-necked round-bottom flask under vigorous stirring. One neck was connected to a gas burette filled with water to monitor the volume of the gas evolution while the other neck was used for the introduction of $3 \mathrm{~mL}$ of aqueous $\mathrm{AB}(46.2 \mathrm{mg}, 1 \mathrm{mmol})$. A shaking table was used to control the hydrolysis reaction of sodium borohydride under vigorous shaking $(220 \mathrm{rpm})$ at $25^{\circ} \mathrm{C}$.

2.5. Characterization. X-ray diffraction (XRD) measurements were performed on Rigaku D-max- $\gamma$ A XRD with $\mathrm{Cu}$ $\mathrm{K} \alpha$ radiation, $\lambda=1.54178 \AA$. High resolution transmission electron microscopy (HRTEM, JEM-2010) was applied for determining the detailed microstructure and composition information. The surface area measurements were performed with $\mathrm{N}_{2}$ adsorption/desorption isotherms at liquid nitrogen temperature $(77 \mathrm{~K})$ after dehydration under vacuum at $150^{\circ} \mathrm{C}$ for $12 \mathrm{~h}$ using automatic volumetric adsorption equipment (Micromeritics ASAP2010). The pore volume was calculated by a single point method at $P / P_{0}=0.99$. The composition of the catalysts was analyzed by ICP-AES (Thermo Fisher Scientific, USA).

\section{Results and Discussion}

3.1. Characterizations of the Ru@MIL-101 Catalysts. Figure 2 displays the PXRD patterns of MIL-101 and Ru@MIL-101, respectively. The MIL-101 framework is maintained well during the catalyst preparation. Furthermore, no significant diffraction peaks of $\mathrm{Ru}$ were detected from wideangle powder X-ray diffractions, which might be due to the immobilization of Ru NPs into the pores of MIL-101.

The $\mathrm{N}_{2}$ adsorption-desorption isotherms of MIL-101, 2.5 wt\% Ru@MIL-101, and 3.7wt\% Ru@MIL-101 are shown in Figure 3. The specific areas of MIL-101, 2.5 wt\% Ru@MIL101, and 3.7 wt\% Ru@MIL-101 are $2976 \mathrm{~m}^{2} / \mathrm{g}, 1823 \mathrm{~m}^{2} / \mathrm{g}$, and $1691 \mathrm{~m}^{2} / \mathrm{g}$, respectively. The pore volume of MIL-101, $2.5 \mathrm{wt} \%$ Ru@MIL-101, and 3.7 wt\% Ru@MIL-101 is $1.832 \mathrm{~cm}^{3} \mathrm{~g}^{-1}$, $1.373 \mathrm{~cm}^{3} \mathrm{~g}^{-1}$, and $1.128 \mathrm{~cm}^{3} \mathrm{~g}^{-1}$ (Figure 4), respectively. The decrease in the amount of specific areas and the pore volume 


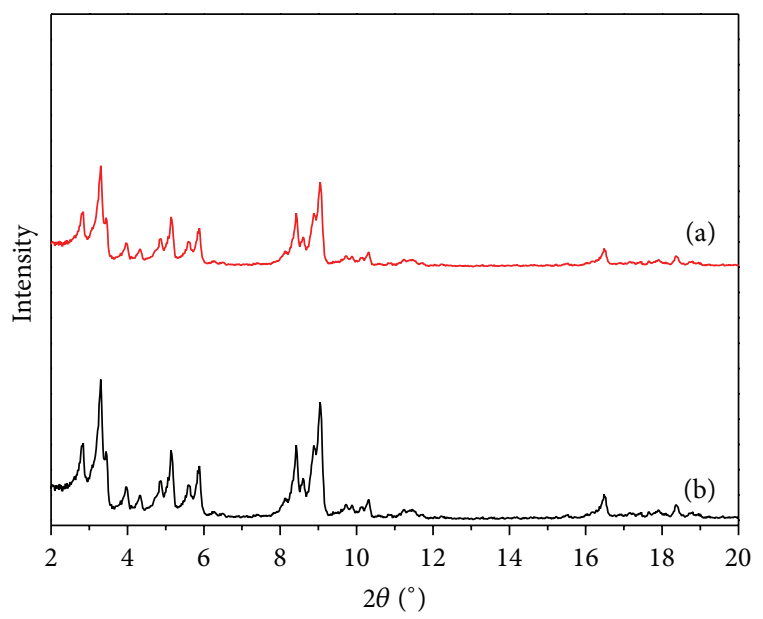

Figure 2: Powder X-ray diffraction patterns of samples (a) Ru@MIL-101 and (b) MIL-101.

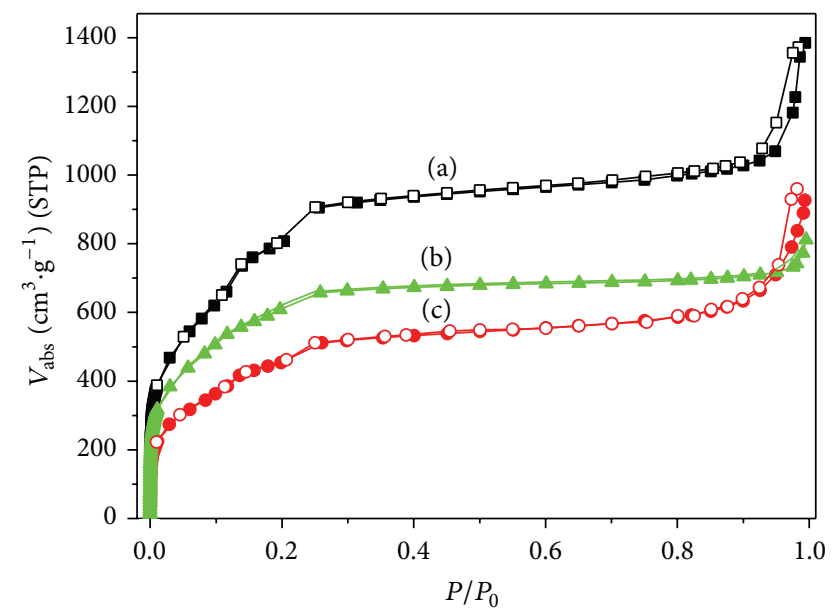

(a) MIL-101

(b) 2.5 wt\% Ru@MIL-101

(c) 3.7 wt\% Ru@MIL-101

Figure 3: Nitrogen sorption isotherms of (a) MIL-101; (b) $2.5 \mathrm{wt} \%$ Ru@MIL-101; (c) 3.7 wt\% Ru@MIL-101 at 77 K. Filled and open symbols represent adsorption and desorption branches, respectively.

indicates that the pores of MIL-101 were occupied by the well dispersed Ru NPs.

Figure 5 shows the HRTEM images of 2.5 wt $\%$ Ru@MIL101 catalyst. HRTEM images (Figures 5(a) and 5(b)) indicate that the $\mathrm{Ru}$ NPs are well dispersed and encapsulated in the cages of the MIL-101. The mean diameter of Ru NPs in Ru@MIL-101 is $2 \pm 0.2 \mathrm{~nm}$ (Figure 5(c)), which is small enough for them to be accommodated in the two mesoporous cavities of MIL-101 (2.9 and $3.4 \mathrm{~nm}$ ). In addition, HRTEM analysis shows that the Ru NPs are crystalline with a spacing distance of $2.16 \AA$ (Figure 5(d)) corresponding to $\mathrm{Ru}(002)$ plane. These results indicate that the ultra small Ru NPs have been effectively immobilized and well dispersed in the pores of MIL-101.

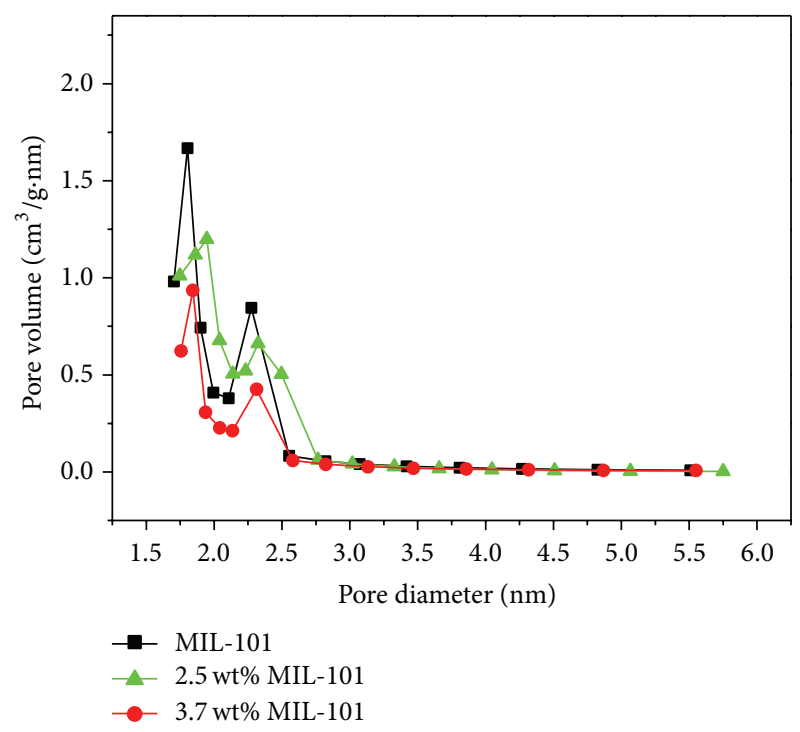

FIGURE 4: The pore diameter distribution of MIL-101, $2.5 \mathrm{wt} \%$ and 3.7 wt\% Ru@MIL-101.

3.2. Catalytic Activity. Figure 6 shows the $\mathrm{H}_{2}$ generation from aqueous $\mathrm{AB}$ under ambient conditions in the presence of Ru@MIL-101. It is found that the $2.5 \mathrm{wt} \%$ and 3.7wt\% Ru@MIL-101 exhibit similar high activity. 2.5 wt\% Ru@MIL-101 catalyst has a high TOF value of $187\left(\mathrm{~mol} \mathrm{H}_{2} \mathrm{~mol}^{-1} \mathrm{Ru} \mathrm{min}^{-1}\right) .3 .7 \mathrm{wt} \%$ Ru@MIL-101 shows no significant increase in TOF value, indicating that the excess $\mathrm{Ru}$ NPs might block the pores and channels of MIL-101. These results are in good agreement with the $\mathrm{N}_{2}$ adsorption-desorption isotherms. This value of TOF is higher than that of Cao et al. [21] using $2.5 \mathrm{wt} \% \mathrm{Ru} / \mathrm{MIL}-101$ $\left(178 \mathrm{~mol} \mathrm{H}_{2} \mathrm{~mol}^{-1} \mathrm{Ru} \mathrm{min}^{-1}\right)$ by solution infiltration method and similar to that of Metin et al. [24] using PSSA-co-MA stabilized $\mathrm{Ru}$ nanoclusters $\left(187.6 \mathrm{~mol} \mathrm{H}_{2} \mathrm{~mol}^{-1} \mathrm{Ru} \mathrm{min}^{-1}\right)$. The same amount of $\mathrm{Ru}$ NPs as the control experiment was reduced by $\mathrm{NaBH}_{4}$ without MIL-101. The release of $\mathrm{H}_{2}$ was slower for $\mathrm{Ru}$ NPs without MIL-101. Moreover, MIL-101 without Ru loading showed no reactivity toward hydrolysis of $\mathrm{AB}$. These results confirm that the Ru@MIL-101 catalysts have cooperative effect on hydrolysis of $\mathrm{AB}$ and excess loading amount of Ru NPs cannot improve the efficiency of the hydrolysis of $\mathrm{AB}$.

The durability of the catalyst is crucial in the practical application. The durability of the $2.5 \mathrm{wt} \%$ Ru@MIL-101 catalyst for hydrolysis of $\mathrm{AB}$ was tested by adding another equivalent of aqueous AB. As shown in Figure 7, even after the 5th run, the catalyst still maintained the original catalytic activity, indicating the Ru NPs have been effectively immobilized in the framework of MIL-101, which can stabilize Ru NPs for good durability.

\section{Conclusion}

In summary, we have successfully prepared a Ru@MIL101 catalyst by immobilizing ultrafine $\mathrm{Ru}$ NPs into the frameworks of MIL-101 using a double solvents method. 


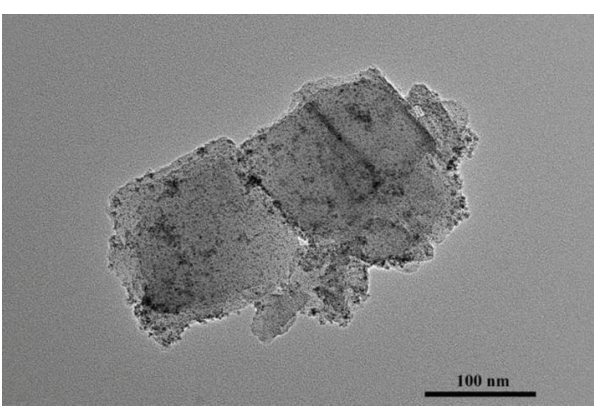

(a)

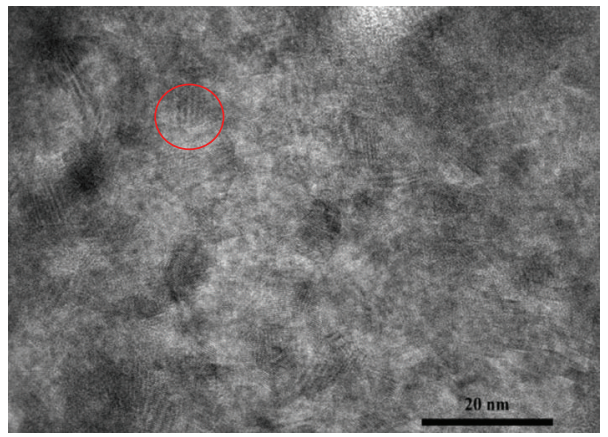

(c)

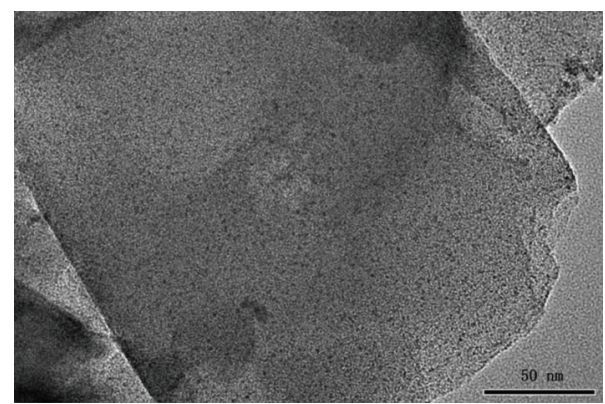

(b)

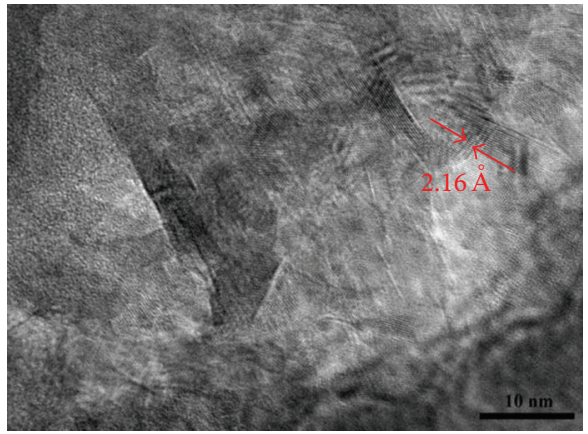

(d)

FIGURE 5: HRTEM images of 2.5 wt\% Ru@MIL-101 catalysts with different magnifications.

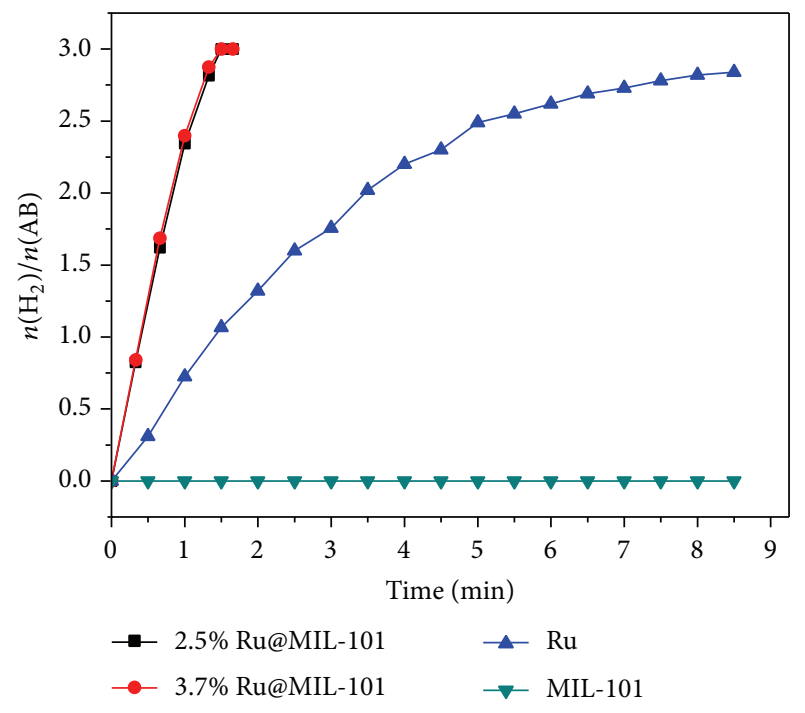

FIgURE 6: Time plots of catalytic dehydrogenation of $\mathrm{AB}$ by $\mathrm{Ru}$, MIL-101, 2.5\% Ru@MIL-101, and 3.7\% Ru@MIL-101.

The synthesized catalyst exhibited highly catalytic activity and excellent durability for catalytic hydrolysis of $\mathrm{AB}$ at ambient conditions. The double solvents approach for the incorporation of Ru MNPs within pores can avoid deposition of the Ru MNPs on the external surface of MIL-101. The combination of high activity and selectivity as well as good durability makes Ru@MIL-101 a potential catalyst for hydrolysis of $A B$ for hydrogen generation. Furthermore, this double

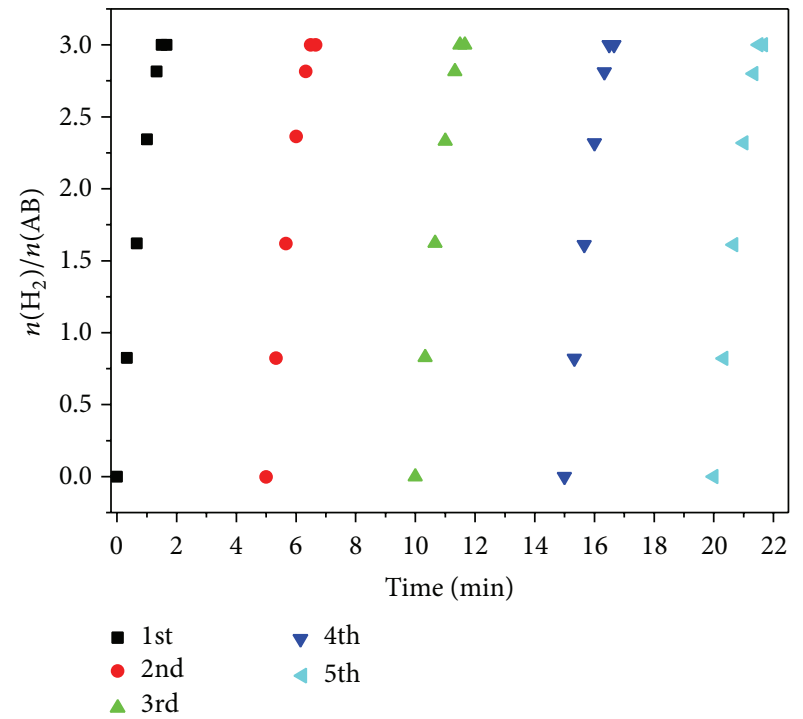

FIGURE 7: Durability test of 2.5 wt\% Ru@MIL-101 for decomposition of $A B$.

solvents method can be extended to other MOFs supported metal nanoparticles for more applications.

\section{Conflict of Interests}

The authors declare that there is no conflict of interests regarding the publication of this paper. 


\section{Acknowledgments}

Financial supports of this research were provided by the scientific research foundation of Shandong province Outstanding Young Scientist Award (no. BS2013NJ017) and the research foundation of Qingdao Science and Technology Development (13-1-4-245-jch).

\section{References}

[1] A. F. Dalebrook, W. Gan, M. Grasemann, S. Moret, and G. Laurenczy, "Hydrogen storage: beyond conventional methods," Chemical Communications, vol. 49, no. 78, pp. 8735-8751, 2013.

[2] L. Schlapbach and A. Züttel, "Hydrogen-storage materials for mobile applications," Nature, vol. 414, no. 6861, pp. 353-358, 2001.

[3] M. Sankir, R. B. Serin, L. Semiz, and N. D. Sankir, "Unusual behavior of dynamic hydrogen generation from sodium borohydride," International Journal of Hydrogen Energy, vol. 39, no. 6, pp. 2608-2613, 2014.

[4] U. Sanyal, U. B. Demirci, B. R. Jagirdar, and P. Miele, "Hydrolysis of ammonia borane as a hydrogen source: fundamental issues and potential solutions towards implementation," ChemSusChem, vol. 4, no. 12, pp. 1731-1739, 2011.

[5] S.-K. Kim, T.-J. Kim, T.-Y. Kim et al., “Tetraglyme-mediated synthesis of Pd nanoparticles for dehydrogenation of ammonia borane," Chemical Communications, vol. 48, no. 14, pp. 20212023, 2012.

[6] R.-Q. Zhong, R.-Q. Zou, T. Nakagawa et al., "Improved hydrogen release from ammonia-borane with ZIF-8," Inorganic Chemistry, vol. 51, no. 5, pp. 2728-2730, 2012.

[7] J.-M. Yan, X.-B. Zhang, S. Han, H. Shioyama, and Q. Xu, "Ironnanoparticle-catalyzed hydrolytic dehydrogenation of ammonia borane for chemical hydrogen storage," Angewandte Chemie International Edition, vol. 47, no. 12, pp. 2287-2289, 2008.

[8] H. Dai, J. Su, K. Hu, W. Luo, and G. Cheng, "Pd nanoparticles supported on MIL-101 as high-performance catalysts for catalytic hydrolysis of ammonia borane," International Journal of Hydrogen Energy, vol. 39, no. 10, pp. 4947-4953, 2014.

[9] M. Chandra and Q. Xu, "Room temperature hydrogen generation from aqueous ammonia-borane using noble metal nanoclusters as highly active catalysts," Journal of Power Sources, vol. 168, no. 1, pp. 135-142, 2007.

[10] Z. Zhang, Y. Zhao, Q. Gong, Z. Li, and J. Li, "MOFs for $\mathrm{CO}_{2}$ capture and separation from flue gas mixtures: the effect of multifunctional sites on their adsorption capacity and selectivity," Chemical Communications, vol. 49, no. 7, pp. 653-661, 2013.

[11] S. Liu, L. Sun, F. Xu et al., "Nanosized Cu-MOFs induced by graphene oxide and enhanced gas storage capacity," Energy \& Environmental Science, vol. 6, no. 3, pp. 818-823, 2013.

[12] B. Liu, W.-P. Wu, L. Hou, and Y.-Y. Wang, "Four uncommon nanocage-based Ln-MOFs: highly selective luminescent sensing for $\mathrm{Cu}^{2+}$ ions and selective $\mathrm{CO}_{2}$ capture," Chemical Communications, vol. 50, no. 63, pp. 8731-8734, 2014.

[13] Y.-N. Wu, M. Zhou, S. Li et al., "Magnetic metal-organic frameworks: $\gamma-\mathrm{Fe}_{2} \mathrm{O}_{3} @ \mathrm{MOF}$ via confined in situ pyrolysis method for drug delivery," Small, vol. 10, no. 14, pp. 2927-2936, 2014.

[14] D. Esken, S. Turner, O. I. Lebedev, G. Van Tendeloo, and R. A. Fischer, "Au@ZIFs: Stabilization and encapsulation of cavity-size matching gold clusters inside functionalized zeolite imidazolate frameworks, ZIFs," Chemistry of Materials, vol. 22, no. 23, pp. 6393-6401, 2010.

[15] H. Li, Z. Zhu, F. Zhang et al., "Palladium nanoparticles confined in the cages of MIL-101: an efficient catalyst for the one-pot indole synthesis in water," ACS Catalysis, vol. 1, no. 11, pp. 16041612, 2011.

[16] H.-L. Jiang, T. Akita, T. Ishida, M. Haruta, and Q. Xu, "Synergistic catalysis of Au@Ag core-shell nanoparticles stabilized on metal-organic framework," Journal of the American Chemical Society, vol. 133, no. 5, pp. 1304-1306, 2011.

[17] C. Wang, K. E. Dekrafft, and W. Lin, “Pt nanoparticles@photoactive metal-organic frameworks: efficient hydrogen evolution via synergistic photoexcitation and electron injection," Journal of the American Chemical Society, vol. 134, no. 17, pp. 7211-7214, 2012.

[18] H.-L. Jiang, B. Liu, T. Akita, M. Haruta, H. Sakurai, and Q. Xu, "Au@ZIF-8: CO oxidation over gold nanoparticles deposited to metal-organic framework," Journal of the American Chemical Society, vol. 131, no. 32, pp. 11302-11303, 2009.

[19] A. Aijaz, A. Karkamkar, Y. J. Choi et al., "Immobilizing highly catalytically active Pt nanoparticles inside the pores of metalorganic framework: a double solvents approach," Journal of the American Chemical Society, vol. 134, no. 34, pp. 13926-13929, 2012.

[20] J. Li, Q.-L. Zhu, and Q. Xu, "Highly active AuCo alloy nanoparticles encapsulated in the pores of metal-organic frameworks for hydrolytic dehydrogenation of ammonia borane," Chemical Communications, vol. 50, no. 44, pp. 5899-5901, 2014.

[21] N. Cao, T. Liu, J. Su, X. Wu, W. Luo, and G. Cheng, "Ruthenium supported on MIL-101 as an efficient catalyst for hydrogen generation from hydrolysis of amine boranes," New Journal of Chemistry, vol. 38, no. 9, pp. 4032-4035, 2014.

[22] S. Wu, L. Chen, B. Yin, and Y. Li, “'Click' post-functionalization of a metal-organic framework for engineering active single-site heterogeneous $\mathrm{Ru}(\mathrm{III})$ catalysts," Chemical Communications, vol. 51, no. 48, pp. 9884-9887, 2015.

[23] J. Hermannsdörfer and R. Kempe, "Selective palladium-loaded MIL-101 catalysts," Chemistry-A European Journal, vol. 17, no. 29, pp. 8071-8077, 2011.

[24] Ö. Metin, Ş. Şahin, and S. Özkar, "Water-soluble poly(4styrenesulfonic acid-co-maleic acid) stabilized ruthenium(0) and palladium(0) nanoclusters as highly active catalysts in hydrogen generation from the hydrolysis of ammonia-borane," International Journal of Hydrogen Energy, vol. 34, no. 15, pp. 6304-6313, 2009. 

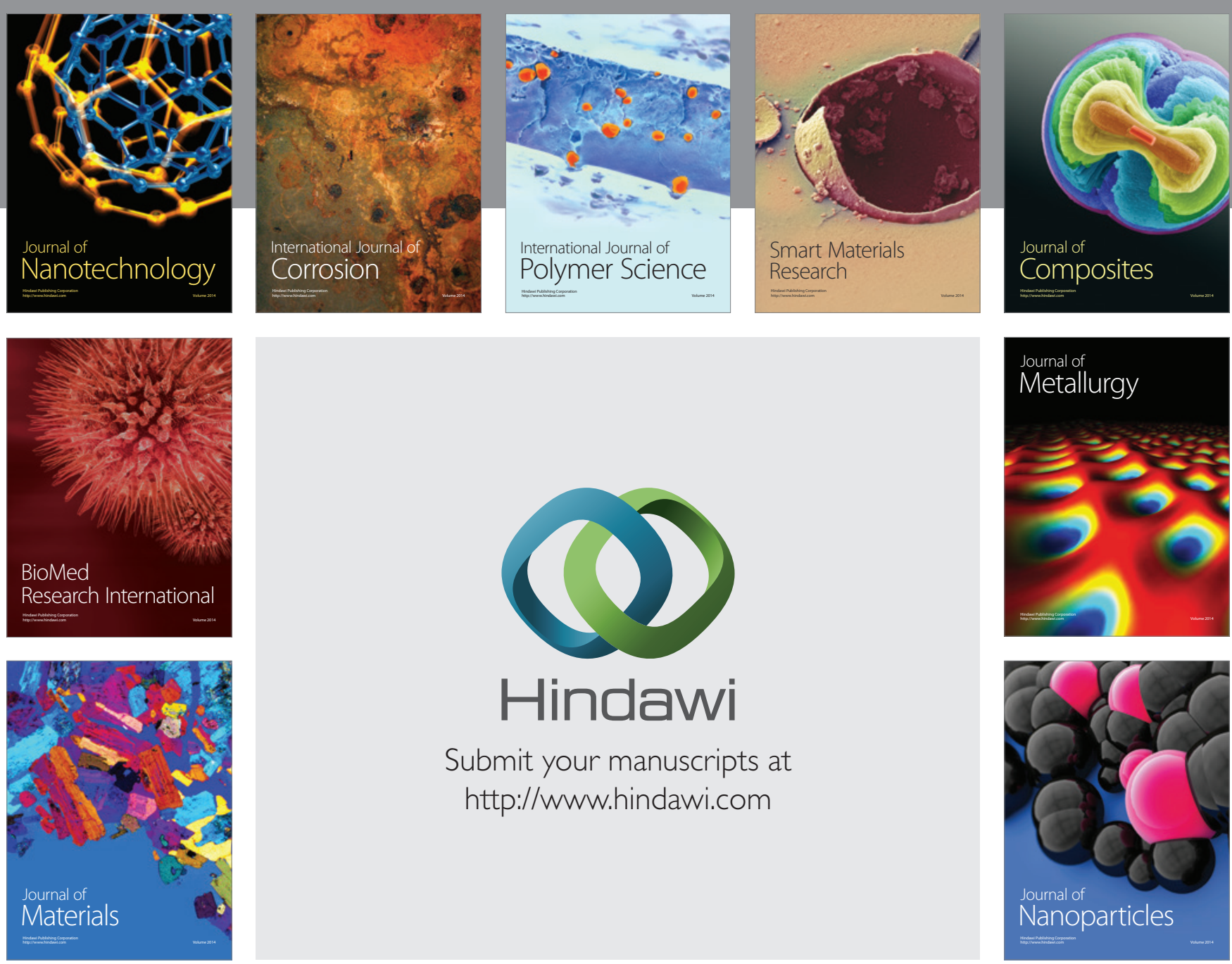

Submit your manuscripts at http://www.hindawi.com
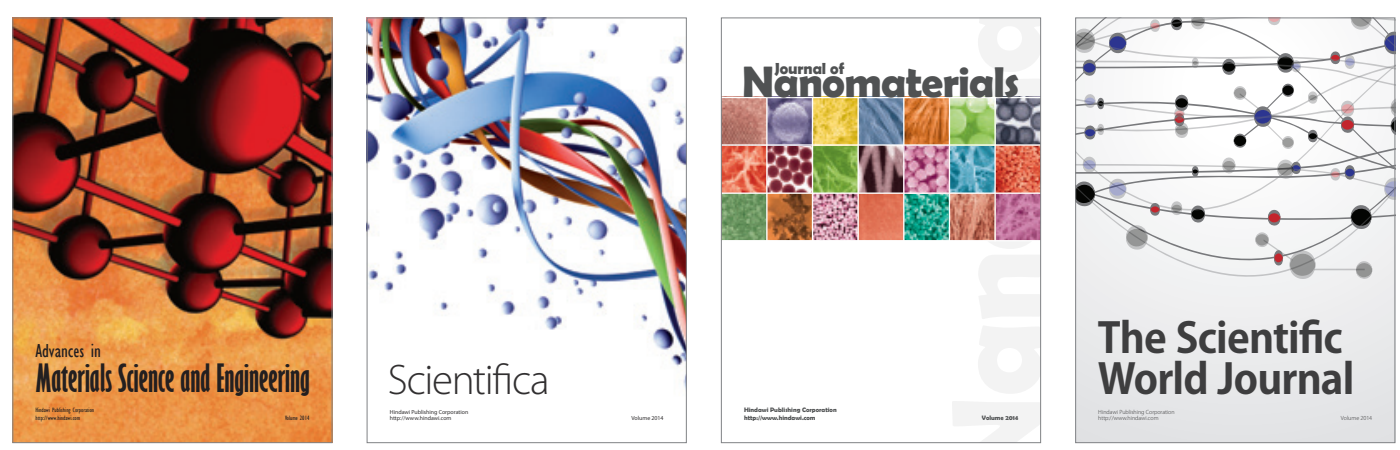

\section{The Scientific World Journal}
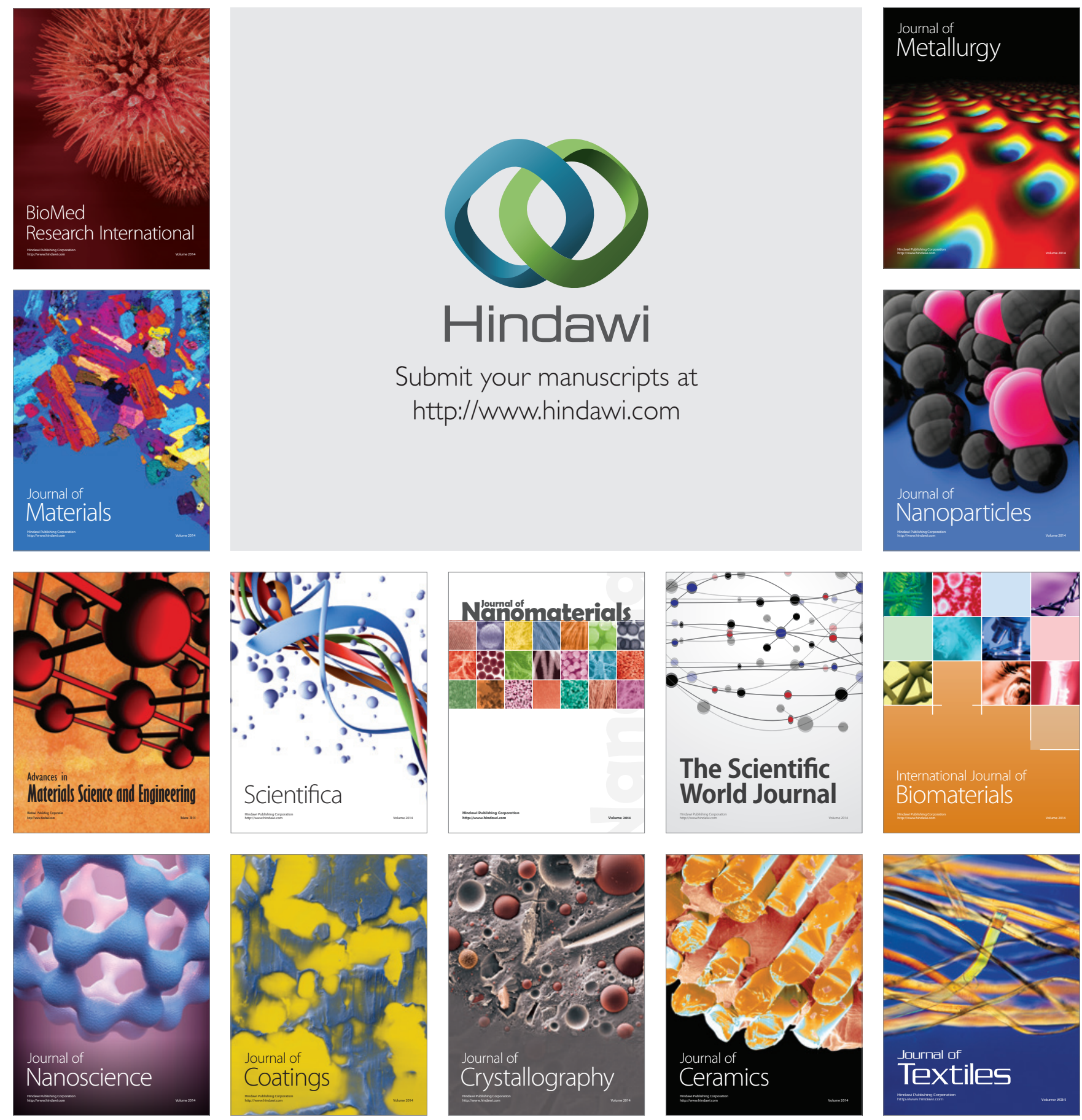